\title{
Desenvolvimento embrionário, larval e juvenil de Hemisorubim platyrhynchos (Siluriformes, Pimelodidae) da bacia do rio Paraná
}

\author{
Fernanda F. Andrade ${ }^{1}$, Maristela C. Makrakis ${ }^{1}$, Ariane F. de Lima ${ }^{1}$, Lucileine de Assumpção ${ }^{1,2}$, \\ Sergio Makrakis ${ }^{1} \&$ Suelen F. R. Pini ${ }^{1}$
}

\begin{abstract}
1. Pós-Graduação em Recursos Pesqueiros e Engenharia de Pesca, Universidade Estadual do Oeste do Paraná - UNIOESTE, Grupo de Pesquisa em Tecnologia de Produção e Conservação de Recursos Pesqueiros e Hídricos - GETECH, Rua da Faculdade, 645, 85903-000, Toledo, PR. (fernandinhaf.andrade@hotmail.com)
\end{abstract}

2. Instituto Água Viva, Rua da Faculdade, 645, 85903-000, Toledo, PR.

\begin{abstract}
Embrionic, larval and juvenile development of Hemisorubim platyrhynchos (Siluriformes, Pimelodidae) from the Paraná River basin. This study aimed to describe the initial stages of development of the Pothole Shovelnose Catfish, Hemisorubim platyrhynchos (Valenciennes, 1840), as well as changes in the allometric growth pattern during early ontogeny. A total of 90 eggs 210 larvae, and 24 juveniles from hatching were analyzed for morphometric and meristic variables, and for besides the allometric growth coefficient in relation to head, trunk, and tail during larval and initial juvenile period. The eggs presented $1.74 \mathrm{~mm}$ of mean diameter; ample vitelline space $(21.29 \%)$ with mean of $0.37 \mathrm{~mm}$; and the average diameter of the yolk was $1.08 \mathrm{~mm}$. The standard length (SL) of the larvae ranged from 3.47 to $11.85 \mathrm{~mm}$, with most measurements a presenting proportional increase throughout development. The total number of myomeres ranged from 40 to 46 (pre-anal=15-17 and post-anal=24-30). The early larvae of $H$. platyrhynchos present pigmentation in the head and in the anteroventral region of the body (anterior and posterior regions of the yolk sac). The pigmentation intensifies in the post-flexion stage along the dorsal region of the head, forming a longitudinal strip extending from the snout to the operculum, as a transversal strip that goes from one side to the other, passing through the anterior region of the dorsal fin, with maculae distributed throughout the body in juveniles $(\mathrm{SL}=19.5-49.09 \mathrm{~mm}$ ). In the early stages of larval development, the head and tail grow much faster than the trunk, indicating priorities related to food and swimming, which tend to isometry, with fast growth of the trunk in early juveniles.
\end{abstract}

KEYWORDS. Early development, morphometry, allometric growth, fish larvae.

RESUMO. O objetivo deste estudo foi descrever o desenvolvimento embrionário, larval e juvenil da jurupoca, Hemisorubim platyrhynchos (Valenciennes, 1840), bem como as mudanças nos padrões de crescimento alométrico durante a ontogenia inicial da espécie. Um total de 90 ovos, 210 larvas e 24 juvenis provenientes de reprodução induzida foram analisados quanto a variáveis morfométricas e merísticas, além do coeficiente de crescimento alométrico em relação à cabeça, tronco e cauda durante o período larval e juvenil inicial. Os ovos apresentaram diâmetro médio de $1,74 \mathrm{~mm}$, espaço perivitelino amplo (21,29\%), com média de $0,37 \mathrm{~mm}$, e diâmetro médio do vitelo de $1,08 \mathrm{~mm}$. O comprimento padrão (CP) das larvas variou de 3,47 a $11,85 \mathrm{~mm}$, com a maioria das medidas apresentando aumento proporcional ao longo do desenvolvimento. O número total de miômeros variou de 40 a 46 (pré-anal=15-17 e pós-anal=24-30). As larvas iniciais de $H$. platyrhynchos apresentam pigmentação na cabeça e na região ântero-ventral do corpo (anterior e posterior do saco vitelino). No estágio de pós-flexão, a pigmentação se intensifica, distribuindo-se na região dorsal da cabeça, formando uma faixa longitudinal que se estende do focinho ao opérculo, assim como uma faixa transversal, de um flanco a outro, passando pela região anterior da nadadeira dorsal, com máculas distribuídas ao longo do corpo nos juvenis $(\mathrm{CP}=19,5-49,09 \mathrm{~mm})$. Nos primeiros estágios de desenvolvimento larval, a cabeça e a cauda crescem muito mais rapidamente do que o tronco, o que indica prioridades relacionadas à alimentação e natação, as quais posteriormente tendem à isometria, com um crescimento rápido do tronco nos juvenis iniciais.

PALAVRAS-CHAVE. Desenvolvimento inicial, morfometria, crescimento alométrico, larvas de peixes.

O entendimento da dinâmica reprodutiva da comunidade íctica permite estabelecer áreas e períodos de desova em ambientes naturais e uma das maneiras para a determinação destas são os estudos de ovos e larvas. A identificação precisa das áreas e períodos de desova tem importância fundamental para a implementação de medidas de preservação (BialetZKi et al., 2001; Fuiman, 2002). Além disso, os estudos de ovos e larvas de peixes subsidiam os estudos de biologia, sistemática, de avaliação de estoques pesqueiros e de dinâmica das populações (Hempel, 1973).

Os caracteres morfológicos, morfométricos e merísticos, pigmentação e estágio de desenvolvimento de estruturas corpóreas, relativos ao tamanho, são parâmetros base para a identificação de larvas de peixes (ARAÚJo-LimA \& DonALD, 1988). A caracterização do desenvolvimento inicial de peixes é imprescindível para a identificação do ictioplâncton na natureza, pois permite relacionar, com maior precisão, os ovos e larvas coletados com a espécie à qual pertencem (BIALETZKI et al., 2001).

Apesar das dificuldades na identificação do material coletado em ambiente natural (BialetzKi et al., 1998), devido ao fato de larvas e adultos de muitas espécies de peixes divergirem quanto à morfologia, dieta, habitats $\mathrm{e}$ comportamento (LeIs \& TRNSKI, 1989), estes estudos têm crescido no Brasil (NAKATANI et al., 2001). Descrições do desenvolvimento embrionário e/ou larval foram feitas para espécies de peixes de diferentes bacias hidrográficas brasileiras (NASCIMENTO \& AraÚJo-Lima, 1993; NAKATANI et al., 1997, 1998; CAVICCHIOLI et al., 1997; BiALETZKi et al., 1998, 2001, 2008; SAnChEs et al., 1999; ReynalteTATAJe et al., 2001; Luz et al., 2001; Sousa \& Severi, 2002; Reynalte-Tataje et al., 2004; Maciel et al., 2009; Oliveira et al., 2012; Hons et al., 2012).

Hemisorubim platyrhynchos (Valenciennes, 1840), popularmente conhecida como jurupoca, é uma espécie de médio porte, pertencente à família Pimelodidae e tem ocorrência nos grandes rios da América do Sul, nas bacias dos rios Amazonas, Maroni, Orinoco e Paraná (ReIs et al., 2003). A espécie é predadora de microfauna bentônica e de peixes, com uma longevidade de 11,4 anos, podendo 
alcançar até $64 \mathrm{~cm}$ de comprimento (Penha et al., 2004). A primeira maturação sexual ocorre com $30,1 \mathrm{~cm}$ de comprimento, sendo sua desova constatada durante o período de cheias entre dezembro e janeiro (Agostinho et al., 2003, SuzuKi et al., 2004). Além disso, H. platyrhynchos é migradora de longa distância (AgostinHo et al., 2003; MAKRAKIs et al., 2012) e é uma espécie suscetível à degradação ambiental e à pesca predatória (PENHA et al., 2004), o que contribui para o declínio das populações naturais. No Brasil, é considerada uma espécie nobre e de grande valor comercial (Penha et al., 2004; Hoeinghaus et al., 2009).

Apesar de sua ampla distribuição e importâncias comercial e ecológica (Hoeinghaus et al., 2009), pouco se conhece sobre a biologia e ecologia desta espécie (PENHA et al., 2004). Assim, este estudo descreve os estágios iniciais de desenvolvimento de $H$. platyrhynchos, por meio de variáveis morfométricas, merísticas e padrão de pigmentação, buscando preencher lacunas no conhecimento da ontogenia inicial da espécie, fundamentais para subsidiar ações de manejo e conservação.

\section{MATERIAL E MÉTODOS}

O material utilizado neste estudo foi proveniente de reprodução induzida de exemplares de H. platyrhynchos da bacia do alto rio Paraná, realizada na Estação de Piscicultura de Jupiá, pertencente à Companhia Energética de São Paulo (CESP), no município de Castilho, Estado de São Paulo, no ano de 2007. Os espécimes-testemunho foram depositados na Coleção de Peixes do Museu de Zoologia da Universidade Estadual de Londrina (MZUEL 3795).

Após a desova das fêmeas induzidas à reprodução, amostras foram coletadas de acordo com o grau de desenvolvimento de ovos e larvas. Os ovos foram coletados logo após a hidratação, com intervalos de duas horas até a eclosão. As larvas foram coletadas desde a eclosão até a completa absorção do saco vitelino, em intervalos de duas a seis horas. Após este período, larvas e juvenis foram coletados periodicamente a cada doze horas. Ovos, larvas e juvenis foram previamente anestesiados com benzocaína e, posteriormente, fixados em uma solução de formol a 4\% tamponado com carbonato de cálcio.

Os ovos foram classificados de acordo com NAKATANI et al. (2001) nos seguintes estágios de desenvolvimento: a) clivagem inicial - estágio em que ocorre a formação das primeiras células; b) mórula; c) blástula; d) gástrula; e) embrião inicial - estágio em que ocorre a diferenciação do embrião; f) formação da cauda; g) cauda livre - estágio em que a cauda se desprende do vitelo; e h) embrião final - estágio em que o embrião se encontra praticamente formado.

As larvas e juvenis foram separados de acordo com o grau de desenvolvimento, segundo АнцSTRom \& BALL (1954), modificado por NAKATANI et al. (2001), em I) período larval: a) estágio larval vitelino - compreende desde a eclosão até quando as larvas apresentam olho completo ou parcialmente pigmentado, abertura do ânus e da boca; b) estágio de pré-flexão - se estende desde a abertura do ânus e da boca até o início da flexão da notocorda, com o aparecimento dos primeiros elementos de suporte da nadadeira caudal; c) estágio de flexão - se caracteriza pelo início da flexão da notocorda com o aparecimento dos elementos suporte da nadadeira caudal até a completa flexão da mesma e aparecimento do botão da nadadeira pélvica e d) estágio de pós-flexão - da completa flexão da notocorda e aparecimento do botão da nadadeira pélvica até a completa formação dos raios de todas as nadadeiras; e II) período juvenil, em que apresentam completa formação dos raios de todas as nadadeiras até a primeira maturação sexual.

As medidas morfométricas, expressas em $\mathrm{mm}$, foram realizadas em microscópico estereoscópio equipado com ocular micrométrica. Dos ovos foram obtidas as seguintes variáveis: diâmetro total (DO); diâmetro do saco vitelino (DSV) e espaço perivitelino (EP) (Fig. 1), considerando que o tamanho do espaço perivitelino foi caracterizado conforme sua participação no volume total do ovo (NAKATANi et al., 2001).

Para as larvas e juvenis iniciais, os caracteres morfométricos obtidos foram: comprimento total (CT); comprimento padrão $(\mathrm{CP})$; comprimento do focinho $(\mathrm{CF})$; diâmetro do olho (DOL); comprimento da cabeça (CC); comprimento do tronco (Ctr); comprimento da cauda (Cca); altura da cabeça (AC); altura do corpo (ACO); distância pré-peitoral (DFNP); distância pré-pélvica (DFNPv); distância pré-dorsal (DFND) e distância pré-anal (DFNA). Os caracteres merísticos foram número de miômeros préanais (NMPA), número de miômeros pós-anais (NMPS) e número de raios das nadadeiras peitoral (NRNP), dorsal (NRND), pélvicas (NRNPv) e anal (NRNA) (Figs 2, 3).

Além da descrição do grau de desenvolvimento de cada período (embrionário, larval e juvenil inicial), foi também observada a ocorrência dos principais eventos morfológicos (fomação da cauda, absorção do saco vitelino, formação da boca, olhos, flexão da notocorda, formação das nadadeiras) e padrão de pigmentação, sendo fotografados e ilustrados os indivíduos que melhor representaram essas características, com auxílio de câmera digital e câmara clara acopladas ao microscópio estereoscópico.

As relações corporais para a altura do corpo, comprimento da cabeça e diâmetro do olho foram estabelecidas utilizando-se os critérios sugeridos por Leis \& TRnski (1989), modificado por NAKATANi et al. (2001). Na análise das relações corporais, as variáveis morfométricas foram expressas como porcentagens do comprimento padrão e comprimento da cabeça ao longo do desenvolvimento: altura do corpo, distância pré-peitoral, distância pré-pélvica, distância pré-dorsal e distância préanal foram relacionadas com o comprimento padrão e o diâmetro do olho, comprimento do focinho e altura da cabeça foram relacionadas com o comprimento da cabeça.

O coeficiente de crescimento alométrico em relação à cabeça, ao tronco e à cauda em proporção ao comprimento total foi calculado para evidenciar as mudanças que ocorrem 

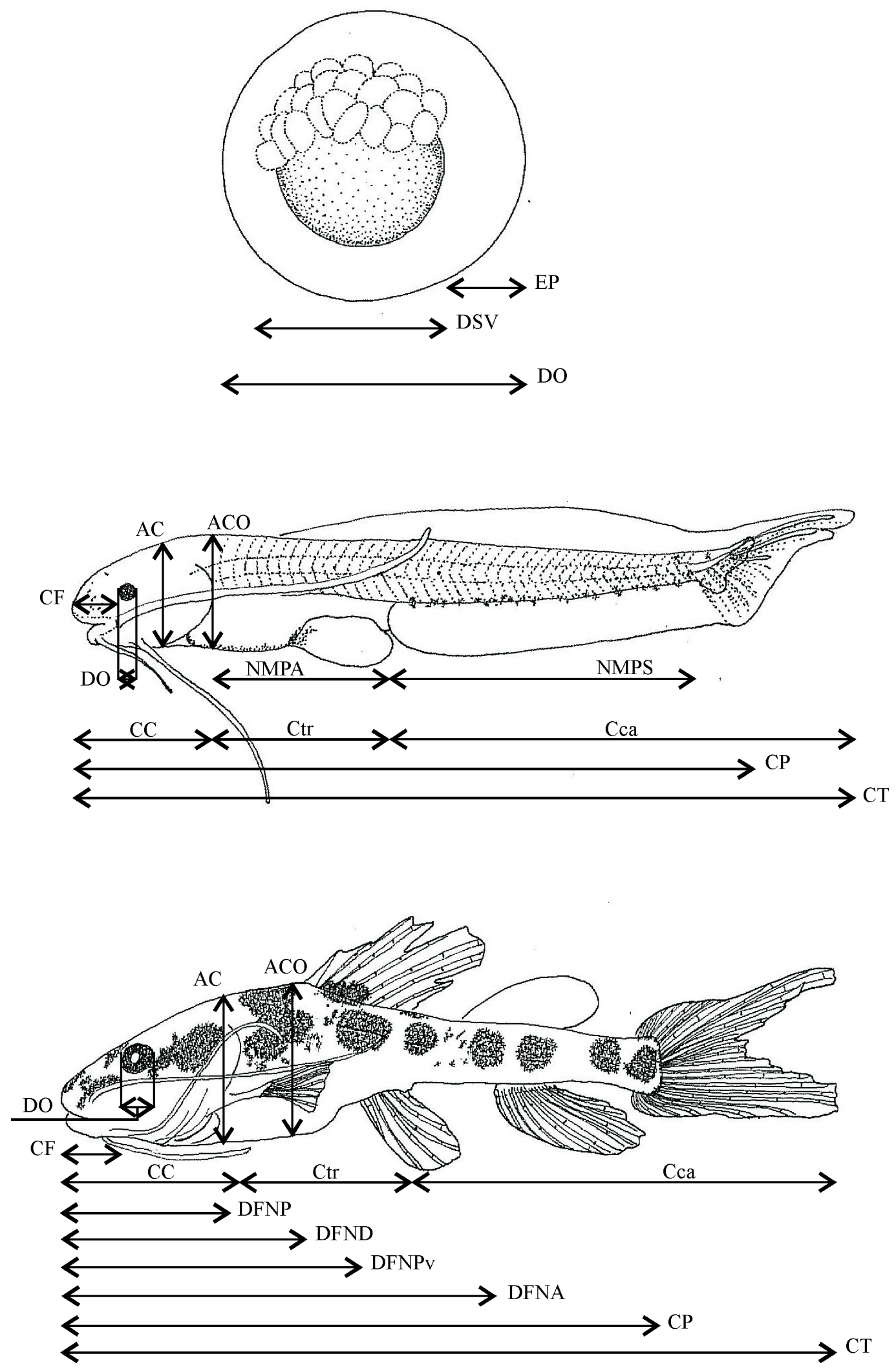

Figs 1-3. Medidas morfométricas realizadas em Hemisorubim platyrhynchos (Valenciennes, 1840): 1, ovos; 2, larvas; 3, juvenis (AC, altura da cabeça; ACO, altura do corpo; CC, comprimento da cabeça; Cca, comprimento da cauda; $\mathrm{CF}$, comprimento do focinho; CP, comprimento padrão; CT, comprimento total; Ctr, comprimento do tronco; DFNA, distância pré-anal; DFND, distância pré-dorsal; DFNP, distância pré-peitoral; DFNPv, distância pré-pélvica; DO, diâmetro total; DOL, diâmetro do olho; DSV, diâmetro do saco vitelino; EP, espaço perivitelino; NMPA, número de miômeros pré-anais; NMPS, número de miômeros pós-anais; NRNA, número de raios das nadadeiras anal; NRND, número de raios das nadadeiras dorsal; NRNP, número de raios das nadadeiras peitoral; NRNPv, número de raios das nadadeiras pélvicas). 
durante o crescimento e desenvolvimento em sucessivos estágios. Os padrões de alometria foram descritos pelo coeficiente de crescimento através da regressão linear simples $\left(\mathrm{Y}=a+\mathrm{X}^{b}\right)$ realizada sobre os dados logtransformados, onde $\mathrm{Y}$ é a variável dependente (caractere medido), $\mathrm{X}$ é a variável independente (comprimento total), $a$ é o intercepto e $b$ é o coeficiente de crescimento (FUIMAN, 1983). Quando $b=1$ tem-se um crescimento isométrico, se $b>1$, o crescimento é alométrico positivo e se $b<1$, o crescimento é alométrico negativo.

\section{RESULTADOS}

Período embrionário. Um total de 90 ovos de $H$. platyrhynchos (Tab. I) foi analisado. Os ovos são esféricos, transparentes e não adesivos, apresentando diâmetro médio de $1,74 \mathrm{~mm}$, diâmetro médio do vitelo de $1,08 \mathrm{~mm}$ e espaço perivitelino amplo $(21,29 \%)$ com média de 0,37 $\mathrm{mm}$. Decorridas quatro horas após a fecundação, iniciase a diferenciação do embrião e a extremidade caudal se desprende depois de aproximadamente 8 horas (Figs 4-11). A duração do período embrionário (incubação - da fertilização à eclosão) correspondeu a 16 horas.

Período larval. As larvas de $H$. platyrhynchos eclodiram 16 horas após a fecundação, com um comprimento padrão médio de $3,83 \pm 0,22 \mathrm{~mm}$, a uma temperatura média de $29,2^{\circ} \mathrm{C}$. Um total de 210 larvas foi analisado, com comprimento padrão variando de 3,47 a 11,85 mm (Tab. I). No estágio larval vitelino (Fig. 12), as larvas apresentam um saco vitelino com formato elíptico e relativamente grande, pigmentação restrita apenas à região ventral do corpo e de forma concentrada nas regiões anterior e posterior do saco vitelino, além de alguns cromatóforos puntiformes na região frontal da cabeça. O olho é evidente, mas pouco pigmentado, e a nadadeira embrionária ("finfold") iniciase na extremidade posterior do saco vitelino, contorna o pedúnculo caudal e segue dorsalmente em direção à cabeça até o nível do saco vitelino. A notocorda é bem visível e o número de miômeros totais nesse estágio varia de 40 a 43 (pré-anal=15-16 e pós-anal=25-27).

No estágio pré-flexão (Fig. 13), o padrão de pigmentação se mantém, os barbilhões maxilares começam a se desenvolver na face lateral da cabeça, o saco vitelino diminui de tamanho e ocorre a formação da boca. O número de miômeros totais varia de 40 a 44 (pré-anal $=15-17$ e pós-anal=24-29).

Na flexão (Figs 14-16), pode-se observar o olho bem pigmentado, a boca e o ânus completamente abertos, o opérculo formado, um par de barbilhões maxilares e dois mentonianos. Ocorre também a flexão da notocorda e a formação dos raios da nadadeira caudal, o saco vitelino é absorvido por completo e os cromatóforos, que se encontravam nas suas extremidades, se tornam mais esparsos e bem distribuídos na região ântero-ventral. No final desse estágio, quando os raios da nadadeira caudal estão completamente formados e segmentados, os raios da nadadeira dorsal começam a se formar, a região ântero-

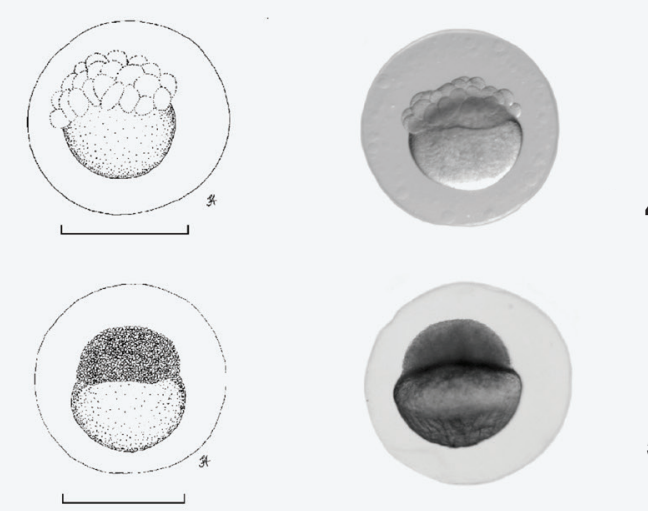

4

5

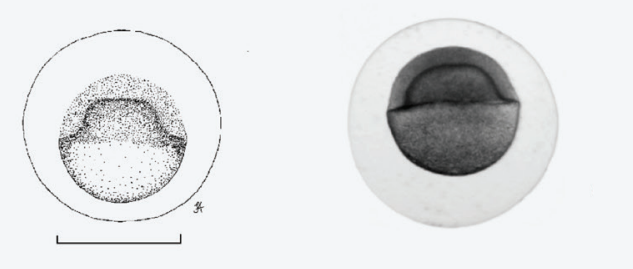

6

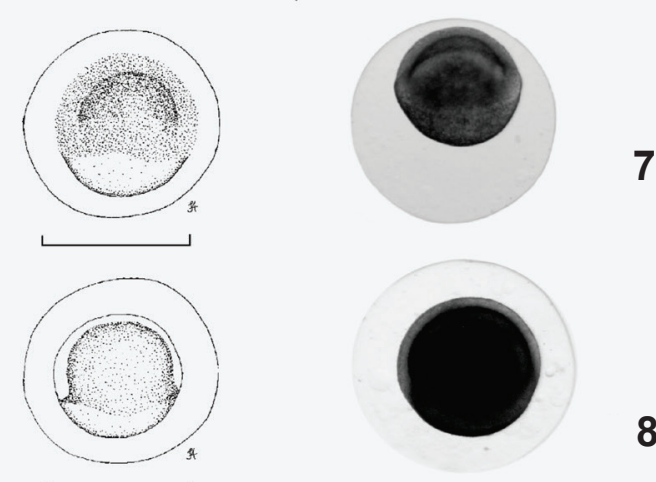

8
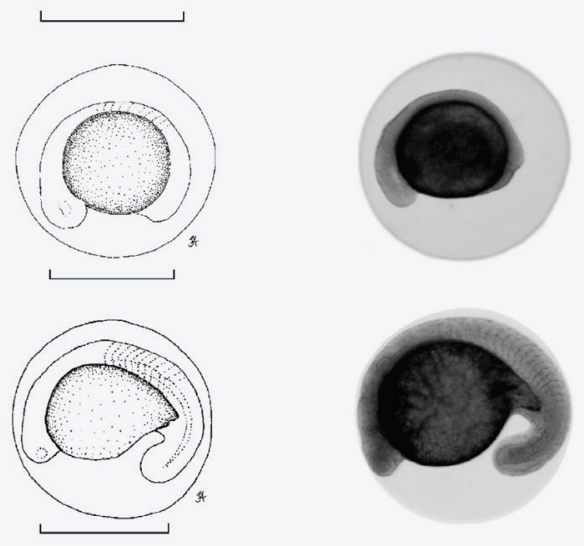

9
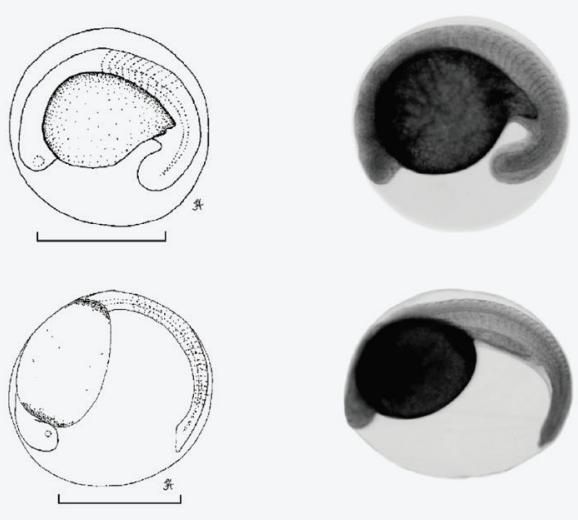

10

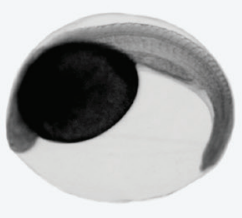

11

Figs 4-11. Desenvolvimento embrionário de Hemisorubim platyrhynchos (Valenciennes, 1840): 4, clivagem inicial; 5, mórula; 6, blástula; 7, gástrula; 8 , embrião inicial; 9 , formação da cauda; 10 , cauda livre; 11, embrião final. Escala $=1 \mathrm{~mm}$. 
Tab. I. Valores máximo (Máx) e mínimo (Mín) e média (X) para as variáveis morfométricas (mm) e merísticas obtidas de ovos, larvas e juvenis de Hemisorubim platyrhynchos (Valenciennes, 1840) (n, número de indivíduos; LV, larval vitelino; PF, pré-flexão; FL, flexão; FP, pós-flexão; J, juvenis; DO, diâmetro total; DSV, diâmetro do saco vitelino; EP, espaço perivitelino; CT, comprimento total; CP, comprimento padrão; CF, comprimento do focinho; DOL, diâmetro do olho; CC, comprimento da cabeça; Ctr, comprimento do tronco; Cca, comprimento da cauda; AC, altura da cabeça; ACO, altura do corpo; NRNP, número de raios da nadadeira peitoral; NRND, número de raios da nadadeira dorsal; NRNPv, número de raios da nadadeira pélvica; NRNA, número de raios da nadadeira anal).

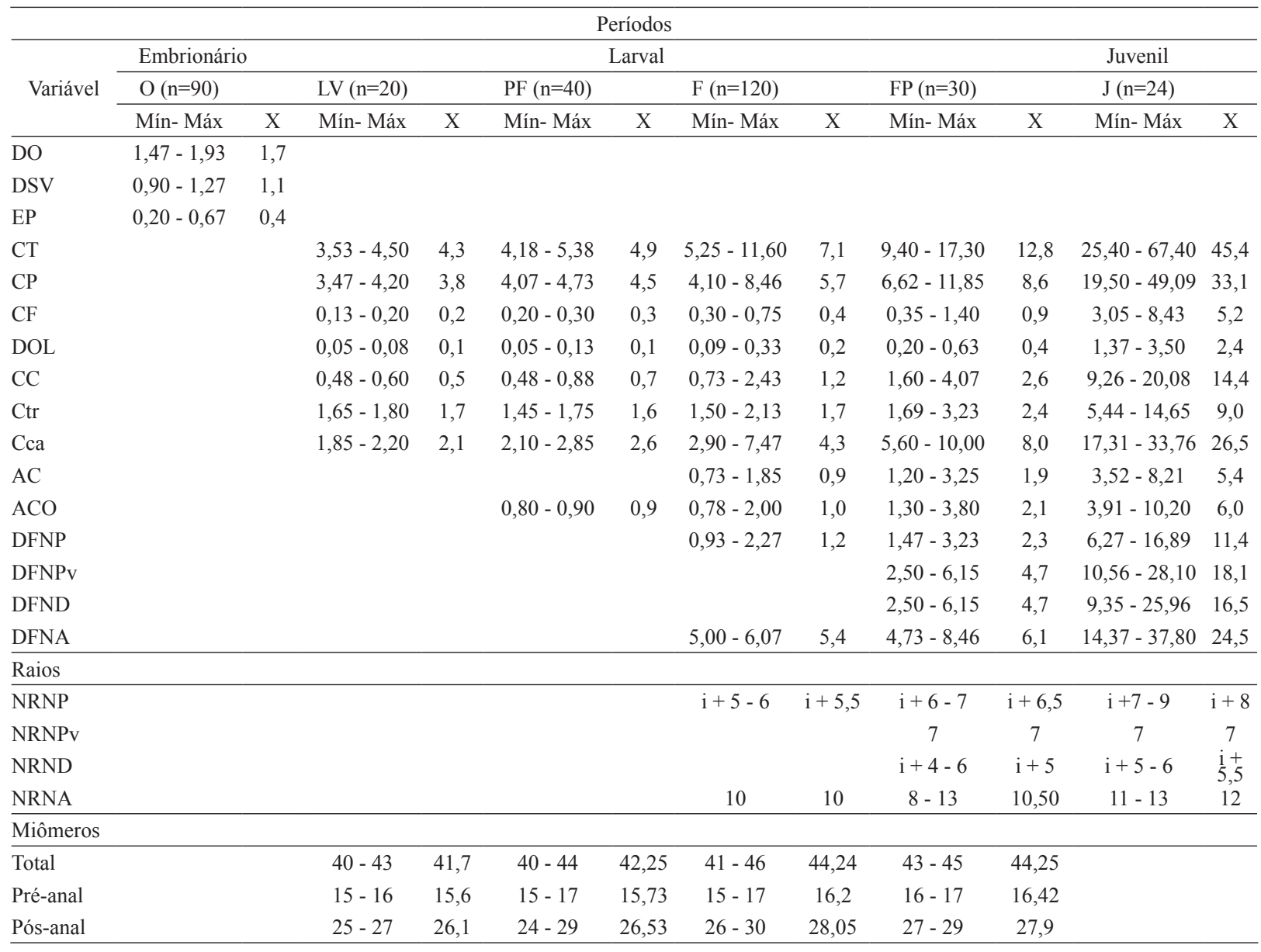

ventral está completamente despigmentada, enquanto na parte superior da cabeça pode-se obervar uma concentração de cromatóforos dendríticos próxima ao olho e ao focinho e na parte lateral do tronco, após o opérculo, formando uma mácula. Nesse estágio, o número de miômeros totais varia de 41 a 46 (pré-anal=15-17 e pós-anal=26-30).

A partir do estágio de pós-flexão (Fig. 17), a pigmentação se intensifica na face lateral da cabeça e também no tronco, após o opérculo, onde forma uma faixa transversal que vai de um flanco a outro, passando pela região anterior da nadadeira dorsal, e também ao longo do corpo, com a presença de aglomerados de cromatóforos dendríticos. As nadadeiras dorsal e anal estão completamente formadas e o botão da nadadeira pélvica pode ser observado. $\mathrm{O}$ número de miômeros totais varia de 43-45 (pré-anal=16-17 e pós-anal=27-29).

Período juvenil. Foram analisados 24 exemplares no período juvenil (Fig. 18), com comprimento-padrão entre 19,5 e 49,09 mm. O número de raios das nadadeiras corresponde a: peitoral i 7 7-9; pélvica 7; dorsal i $+5-6$ e anal 11-13 (Tab. I). Neste período, não foi possível visualizar os miômeros. Os juvenis apresentam pigmentação na cabeça (faixa longitudinal anterior e posterior ao olho), além de máculas, uma no opérculo e outras distribuídas ao longo do corpo, principalmente acima da linha lateral, e na base da nadadeira dorsal. Todos os barbilhões e nadadeiras estão pigmentados.

Relações corporais e alometria. Houve variação nas relações corporais ao longo do desenvolvimento da espécie. A maioria das medidas biométricas apresentou um aumento proporcional ao longo do desenvolvimento, com exceção da altura da cabeça, que apresentou uma diminuição (Figs $19-21,22-27)$. O olho é pequeno $(6,45 \%$ a $21,57 \%)$, a cabeça variou de pequena a grande $(11,13 \%$ a $42,65 \%)$ e o corpo de longo a moderado (13,14\% a $32,08 \%)$.

A relação do crescimento da cabeça, do tronco e da cauda em relação ao comprimento total mostrou-se diferente para os estágios de desenvolvimento larval e período juvenil (Figs 28-32). No estágio larval vitelino, de pré-flexão e flexão (Figs 28, 29, 30, respectivamente) os coeficientes de crescimento da cabeça $(b=1,25 ; 3,01$ e 1,19$)$ e da cauda $(b=1,49 ; 1,77$ e 1,22$)$ mostraram um crescimento 

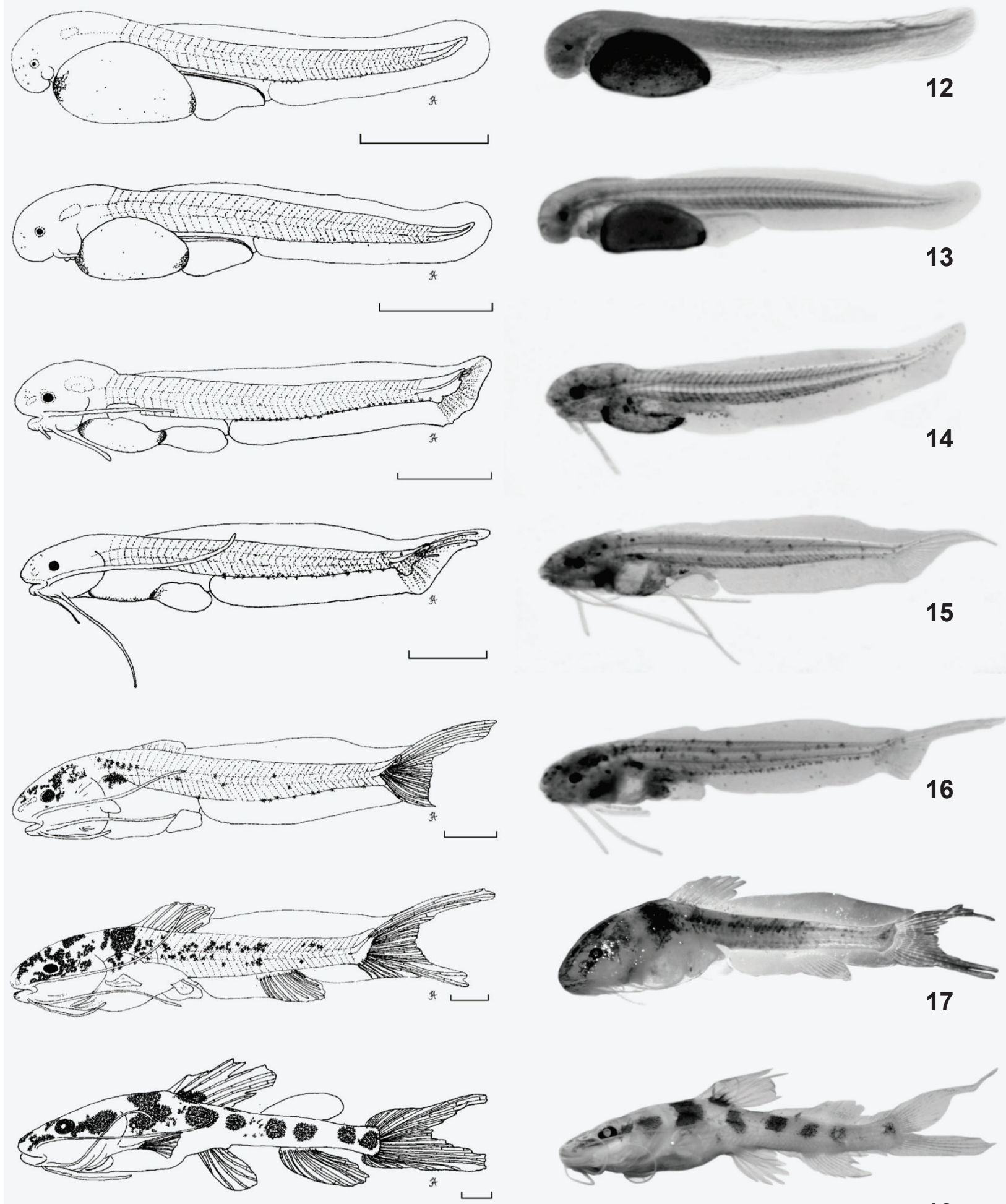

Figs 12-18. Desenvolvimento de larvas e juvenis de Hemisorubim platyrhynchos (Valenciennes, 1840): 12, larval vitelino; 13, pré-flexão; 14, início de flexão; 15, flexão; 16, final de flexão; 17, pós-flexão; 18, juvenil. 

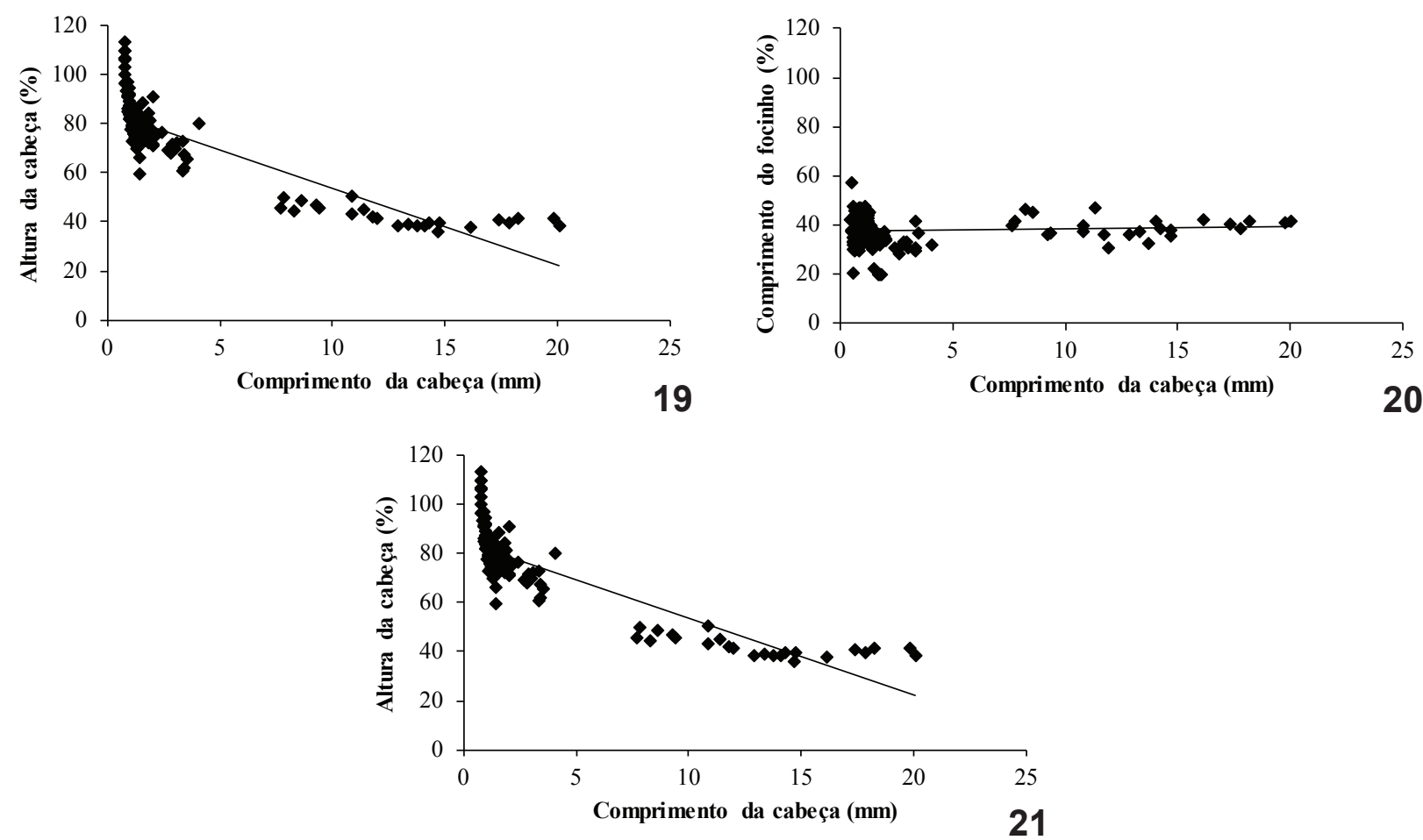

Figs 19-21. Relações corporais de Hemisorubim platyrhynchos (Valenciennes, 1840) obtidas em função do comprimento da cabeça: 19, diâmetro do olho; 20, comprimento do focinho; 21 , altura da cabeça.

alométrico positivo, enquanto o coeficiente de crescimento do tronco $(b=0,33 ;-0,54$ e 0,31$)$, nestes três estágios, mostrou um crescimento alométrico negativo. Na larva em pós-flexão (Fig. 31), a cabeça manteve um crescimento alométrico positivo $(b=1,47)$, o tronco apresentou um crescimento alométrico negativo $(b=0,72)$, enquanto que a cauda apresentou um crescimento isométrico $(b=0,94)$. No período juvenil (Fig. 32), os coeficientes de crescimento da cabeça e da cauda ( $b=1,06$ e 0,86 , respectivamente) mostraram um crescimento isométrico, enquanto o tronco $(b=1,27)$ apresentou um crescimento alométrico positivo.

\section{DISCUSSÃO}

Hemisorubim platyrhynchos apresentou desenvolvimento embrionário com características semelhantes àquelas da maioria dos teleósteos de água doce (CASTELLANi et al., 1994; Ribeiro et al., 1995; NAKATANI et al., 2001). O espaço perivitelino amplo apresentado pela espécie é uma característica comum em espécies migradoras que desovam em ambientes lóticos, como Pseudoplatystoma corruscans (Spix \& Agassiz, 1829) (NAKATANi et al., 2001) e B. orbignyanus (Reynalte-TATAJE et al., 2004) e possivelmente representa um mecanismo adaptativo para garantir a sobrevivência do embrião, diminuindo a ação de choques mecânicos provocados pela correnteza (SANCHES et al., 2001).

As alterações ocorridas no desenvolvimento inicial variam de acordo com a espécie, sendo este desenvolvimento influenciado por vários fatores, dentre eles, a temperatura da água (HuET, 1978). Estudos realizados por Luz et al. (2001) com Pimelodus maculatus Lacépède, 1803 mostraram que a diferenciação do embrião ocorre após $10 \mathrm{~h} 50 \mathrm{~min}$, a uma temperatura de $23,1 \pm 0,5^{\circ} \mathrm{C}$, semelhante ao tempo observado por Hons et al. (2012) para Steindachneridion parahybae (Steindachner, 1897) $\left(23,8 \pm 0,8^{\circ} \mathrm{C}\right)$. Por outro lado, NAKATANi et al. (2001) reportaram diferenciação do embrião de $P$. corruscans a partir das $3 \mathrm{~h} 15$ min e o desprendimento da cauda ocorre após $7 \mathrm{~h}$, enquanto LANDINES et al. (2003) relataram para a mesma espécie o fechamento do blastóporo $5 \mathrm{~h}$ após a fertilização e o desprendimento da cauda após $14 \mathrm{~h}$, com temperatura média de $26,5 \pm 0,4^{\circ} \mathrm{C}$. Os resultados obtidos no presente estudo para $H$. platyrhynchos foram similares aos de $P$. corruscans em relação à diferenciação do embrião e desprendimento da cauda, com pequenas diferenças no tempo de desenvolvimento em relação a outras espécies.

As larvas de $H$. platyrhynchos podem ser consideradas "altriciais". Segundo Fuiman (2002), este tipo de larva é pouco desenvolvida à eclosão, com alguns milímetros de comprimento (comprimento padrão médio de $3,83 \pm 0,22 \mathrm{~mm})$ e transparentes. O saco vitelino é grande, os olhos estão desenvolvidos, porém, sem pigmentos, com boca e brânquias ausentes (FuIMAN, 2002). O pouco desenvolvimento corporal neste estágio está intimamente relacionado às características reprodutivas da espécie (Oliveira et al., 2012), como alta fecundidade e ausência de cuidado parental, as quais possuem rápido desenvolvimento embrionário. Estudos realizados com outras espécies (NAKATANi et al., 2001; Luz et al., 2001; Reynalte-TATAJE $e t$ 

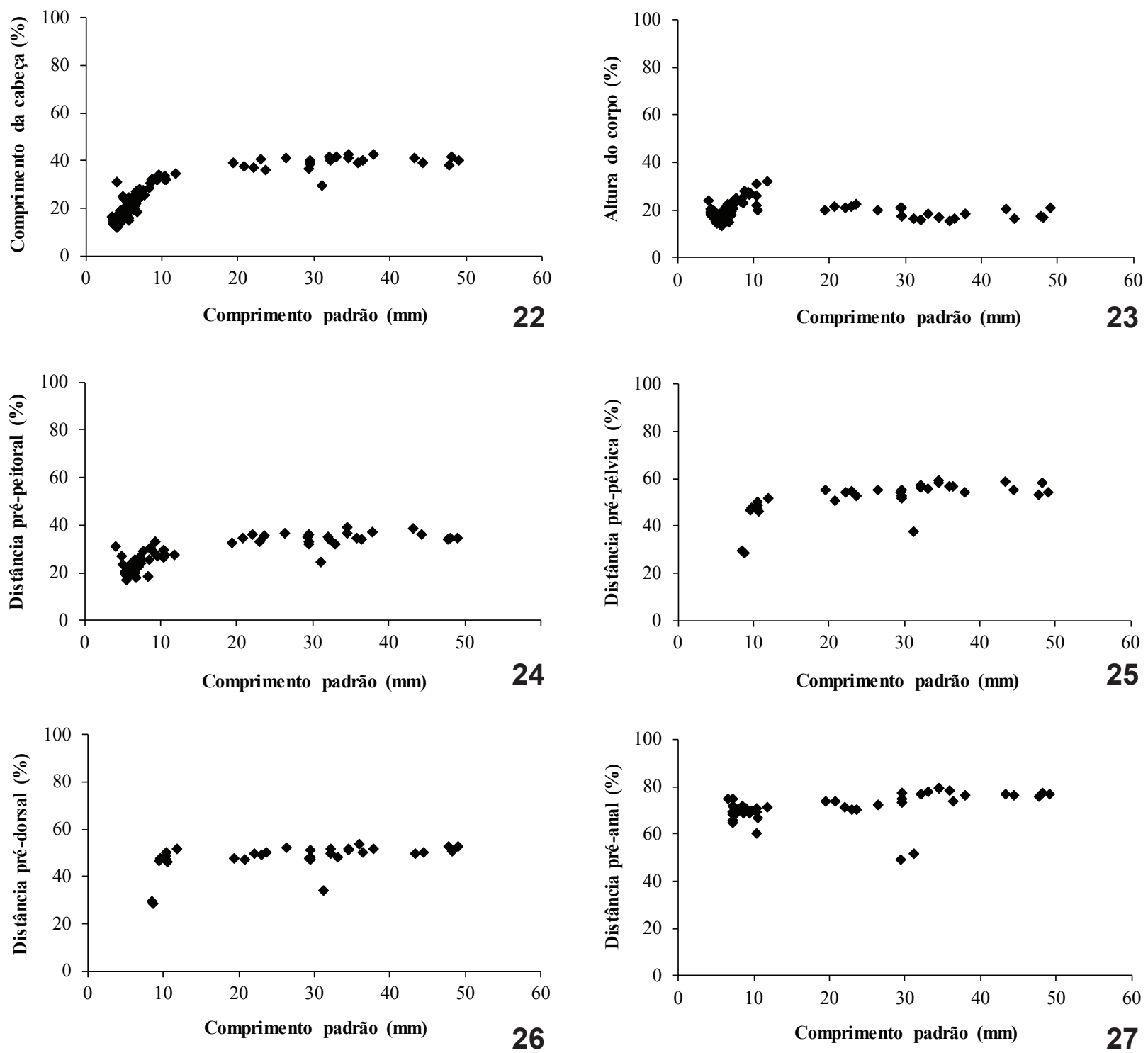

Figs 22-27. Relações corporais de Hemisorubim platyrhynchos (Valenciennes, 1840) obtidas em função do comprimento padrão: 22, comprimento da cabeça; 23, altura do corpo; 24, distância pré-peitoral; 25, distância pré-pélvica; 26, distância pré-dorsal; 27, distância pré-anal.

al., 2004; HonjI et al., 2012; OLIVEIRA et al., 2012) também indicam curto período de incubação com eclosão de larvas caracterizadas por pouco desenvolvimento corporal.

O padrão de pigmentação das larvas pode representar um caractere taxonômico fundamental na identificação de espécies (NASCIMENTO \& ARAúJo-LimA, 1993; Meijide \& Guerrero, 2000; Oliveira et al., 2012). Segundo Kendall et al. (1984), cada espécie tem um padrão distinto de pigmentação, sendo o tamanho e a localização dos melanóforos determinados geneticamente. Em relação ao padrão de pigmentação das larvas de peixes, os cromatóforos podem apresentar forma dendrítica (ARAÚJOLimA, 1985) e puntiforme. As larvas de H. platyrhynchos, nos estágios iniciais, possuem cromatóforos exclusivamente puntiformes, distribuídos na região ventral do corpo, de forma concentrada nas regiões anterior e posterior do saco vitelino e de forma esparsa na parte frontal da cabeça.
As larvas iniciais de $P$. corruscans (NAKATANi et al., 2001) possuem o padrão de pigmentação similar ao de H. platyrhynchos, com exceção da pigmentação presente na cabeça, que pode ser uma característica que diferencie as duas espécies, visto que há uma grande dificuldade na identificação das larvas destas espécies nos primeiros estágios de desenvolvimento.

$\mathrm{O}$ número total ou parcial de miômeros (número total das vértebras menos um) é considerado uma importante variável na identificação de larvas de peixes (SNYDER, 1979), que pode ser uma característica determinante para a separação de espécies, especialmente nos estágios de pré-flexão e flexão (CAVICCHIOLI et al., 1997). Há, porém, uma leve sobreposição do número de miômeros totais entre $H$. platyrhynchos (40 a 46) e $P$. corruscans (45 a 47) (NAKATANi et al., 2001), o que dificulta a separação das espécies, que pertencem a uma mesma família, nos 

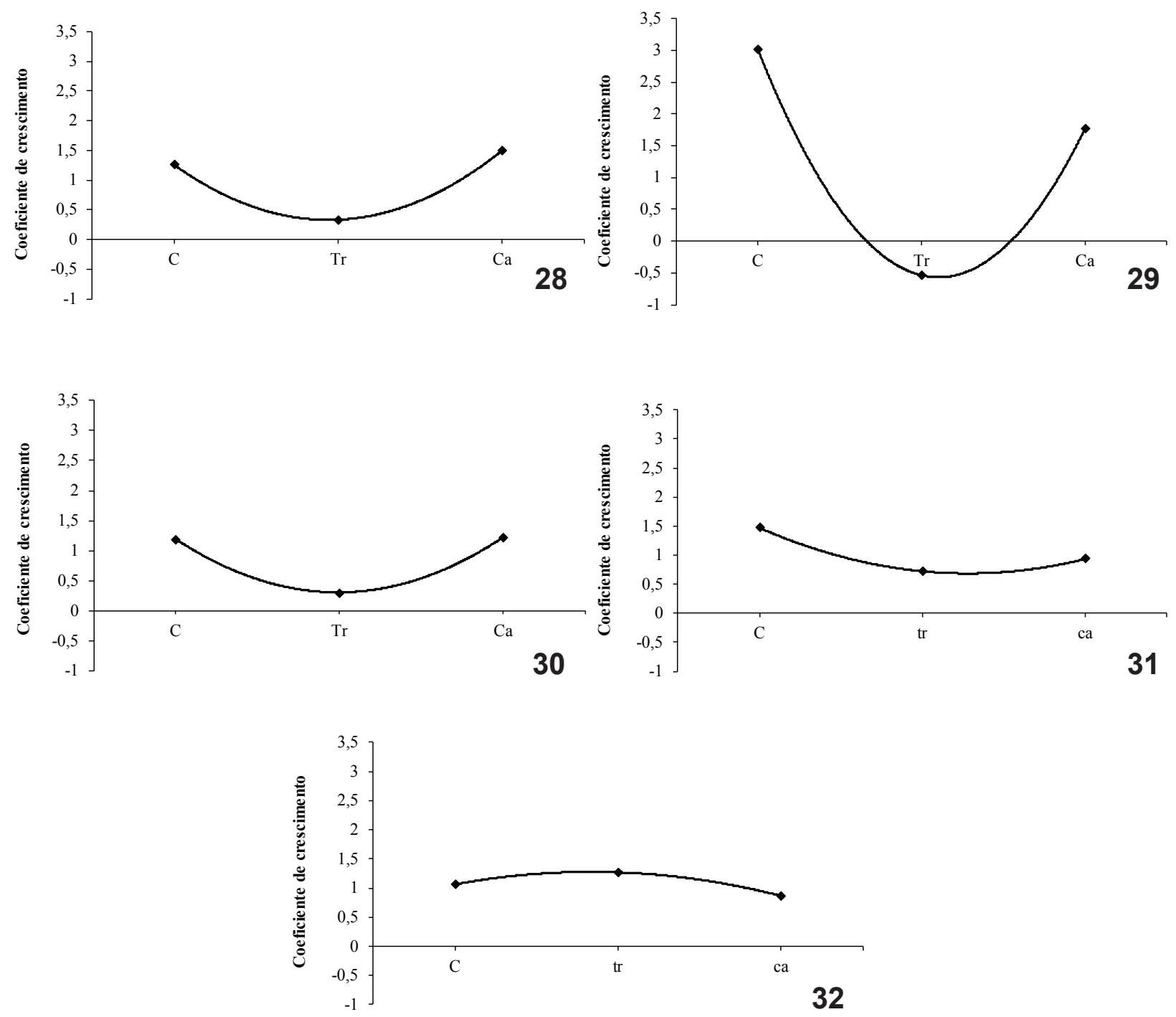

Figs 28-32. Coeficiente de crescimento da cabeça (C), tronco (Tr) e cauda (Ca) em larvas e juvenis de Hemisorubim platyrhynchos (Valenciennes, 1840) para os diferentes estágios de desenvolvimento larval e juvenil: 28 , larval vitelino; 29 , pré-flexão; 30, flexão; 31 , pós-flexão; 32 , juvenil.

estágios iniciais com base neste caractere.

O crescimento alométrico durante o desenvolvimento larval inicial é considerado uma resposta adaptativa para neutralizar pressões ambientais (OsSE \& VAN DEN BOOGARD, 2004), aumentando a probabilidade de sobrevivência e de crescimento durante o desenvolvimento inicial, produzindo alterações na forma corporal devido a um crescimento diferenciado dos órgãos e sistemas envolvidos em funções básicas (ou seja, alimentação, respiração e locomoção) (Fuiman, 1983), ao invés de órgãos que têm menor prioridade para a sobrevivência (OSSE \& VAN DEN BOOGARD, 1995).

As mudanças na forma do corpo e, consequentemente, em desenvolvimento funcional podem ocorrer rapidamente durante desenvolvimento inicial; a partir de um estado de desenvolvimento incipiente na eclosão, processos de diferenciação têm lugar durante o período de nutrição endógena para assegurar o êxito na transição da endógena para exógena (PEÑa \& Dumas, 2009). Um rápido crescimento inicial da cabeça e, na maioria dos casos, um rápido crescimento da cauda, observados para várias espécies de teleósteos (Fuiman, 1983; van SNIK et al., 1997; KOUMOUNDOUROs et al., 1999; GisBert et al., 2002; OSSE \& VAN DEN BOOGARD, 2004), também foi verificado para as larvas de $H$. platyrhynchos nos primeiros estágios do desenvolvimento, indicando necessidade prioritária de crescimento para maximizar a alimentação, escapar de predadores e reduzir o gasto energético durante a locomoção. Lima et al. (2012) também observaram um crescimento acentuado da cabeça inicialmente para Cathorops spixii (Agassiz, 1829), espécie que incuba os ovos na boca, porém na fase de embriões - a larva da espécie eclode bem desenvolvida. Estudos de VAN SNIK et al. (1997) mostraram que, para Cyprinus carpio Linnaeus 
1758 e Clarias gariepinus (Burchell, 1822), essa prioridade inicial de crescimento da cabeça e da cauda desaparece em direção à fase juvenil; o atraso inicial de crescimento do tronco também se reflete na alometria negativa inicial do intestino, o qual é rápido no período subsequente ao desenvolvimento larval. O mesmo padrão de crescimento foi verificado por KaJı et al. (1996, 1999) em estudos com espécies do gênero Thunnus (família Scombridae), como a albacora [Thunnus albacares (Bonnaterre, 1788)] e o atum de barbatana azul [Thunnus thynnus (Linnaeus, 1758)], bem como para H. platyrhynchos no presente estudo, no qual o crescimento acentuado do tronco nos juvenis iniciais pode indicar mudanças na alimentação.

Com o presente estudo pode-se constatar que $H$. platyrhynchos apresenta um desenvolvimento embrionário rápido, suas larvas são pouco desenvolvidas à eclosão, com saco vitelino grande e elíptico. Com o desenvolvimento, ocorrem mudanças no padrão de pigmentação, crescimento inicial acentuado da cabeça e da cauda associados ao desenvolvimento cerebral e da natação e, posteriormente, do tronco nos juvenis iniciais associados à alimentação, que refletem prioridades de desenvolvimento quando órgãos importantes estão se desenvolvendo para a sobrevivência juvenil. As informações obtidas neste trabalho contribuem para a ecologia e sistemática da espécie, particularmente no que se refere à variação ontogênica na morfologia e crescimento, que poderão subsidiar tanto a larvicultura quanto às ações de manejo e conservação da espécie.

Agradecimentos. Os autores agradecem à Companhia Energética de São Paulo - CESP pelo fornecimento do material biológico utilizado no estudo.

\section{REFERÊNCIAS BIBLIOGRÁFICAS}

Agostinho, A. A.; Gomes, L. C.; Suzuki, H. I. \& Júlio-Jr, H. F. 2003. Migratory fishes of the upper Paraná River basin Brazil. In: Carolsfeld, J.; Harvey, B.; Ross, C. \& Baer, A. eds. Migratory fishes of South America: biology, fisheries and conservation status. Vitoria, World Fisheries Trust. p.19-89.

Ahlstrom, E. H. \& Ball, O. P. 1954. Description of eggs and larvae of jack mackerel (Trachurus symmetricus) and distribution and abundance of larvae in 1950 and 1951. United States Department of the Interior Fish and Wildlife Service Fishery Bulletin 56(97):209-245.

Araújo-Lima, C. A. R. M. 1985. Aspectos biológicos de peixes amazônicos. V. Desenvolvimento larval do jaraqui-escama grossa, Semaprochilodus insignis (Characiormes, Pisces) da Amazônia Central. Revista Brasileira de Biologia 45(4):423-431.

Araújo-Lima, C. A. R. M. \& Donald, E. 1988. Número de vértebras de Characiformes e seu uso na identificação de larvas do grupo. Acta Amazonica 18(1-2):351-358.

Bialetzki, A.; Sanches, P. V.; Baumgartner, G. \& Nakatani, K. 1998. Caracterização morfométrica e distribuição temporal de larvas e juvenis de Apareiodon affinis (Steindachner, 1879) (Osteichthyes, Parodontidae) no alto rio Paraná - PR. Revista Brasileira de Zoologia 15(4):1037-1047.

Bialetzki, A.; Sanches, P. V.; Baumgartner, G.; Makrakis, M. C. \& Taguti, T. L. 2008. Desenvolvimento inicial de Hoplias aff. malabaricus (Bloch, 1794) (Osteichthyes, Erythrinidae) da planície alagável do alto rio Paraná, Brasil. Acta Scientiarum, Biological Sciences 30(2): 141-149.

Bialetzki, A.; Baumgartner, G.; Sanches, P. V.; Galuch, A. V.; Luvisuto, M. A.; Nakatani, K.; Makrakis, M. C. \& Borges, M. E. E. 2001. Caracterização do desenvolvimento inicial de Auchenipterus osteomystax (Osteichthyes, Auchenipteridae) da bacia do rio Paraná, Brasil. Acta Scientiarum, Biological Sciences 23(2):377-382.

Castellani, L.; Godinho, H.; Leme dos Santos, H.; Sant, R.; Faria, A. \& SANTOS, M. 1994. Desenvolvimento embrionário do curimbatá Prochilodus lineatus (Valenciennes, 1836) (Cypriniformes, Prochilodontidae). Revista Brasileira de Morfologia 11(2):99-105.

Cavicchioli, M.; Nakatani, K. \& Shibatta, O. A. 1997. Morphometric variation of larvae and juveniles of the piranhas Serrasalmus spilopleura and S. marginatus (Characidae: Serrasalminae) of the Paraná basin, Brazil. Ichthyological Exploration of Freshwaters 8:97-106.

Fuiman, L. A. 1983. Growth gradients in fish larvae. Journal of Fish Biology 23(1):117-123.

2002. Special considerations of fish eggs and larvae. In: Fuiman, L. A. \& WERNER, R. G. eds. Fishery science: the unique contributions of early life stages. United Kingdom, Blackwell Science. p.1-32.

Gisbert, E.; Merino, G.; Muguet, J. B.; Bush, D.; Piedrahita, R. H. \& ConkLin, D. E. 2002. Morphological development and allometric growth patterns in hatchery-reared California halibut larvae. Journal of Fish Biology 61:1217-1229.

Hempel, G. 1973. On the use of ichthyoplankton surveys. FAO Fisheries Technical Paper 122:1-2.

Hoeinghaus, D. J.; Agostinho, A. A.; Gomes, L. C.; Pelicice, F. M.; Okada, E. K.; Latini, J. D.; Kashiwaqui, E. A. L. \& Winemiller, K. O. 2009. Effects of river impoundment on ecosystem services of large tropical rivers: Embodied energy and market value of artisanal fisheries. Conservation Biology 23(5):1222-1231.

Honj, R. M.; Tolussi, C. E.; Mello, P. H.; Caneppele, D. \& Moreira, R. G. 2012. Embryonic development and larval stages of Steindachneridion parahybae (Siluriformes: Pimelodidae): implications for the conservation and rearing of this endangered neotropical species. Neotropical Ichthyology 10:313-327.

Huet, M. 1978. Tratado de Piscicultura. Madri, Mundi- Prensa. 745p.

Kaj,, T.; Tanaka, M.; Oka, M.; Takeuchi, H.; Ohsumi, S.; Teruya, K. \& HiROKAWA, J. 1999. Growth and morphological development of laboratory-reared yelllowfin tuna Thunnus albacares larvae and early juveniles, with special emphasis on the digestive system. Fisheries Science 65(5):700-707.

KaJi, T.; TanaKa, M.; TaKahashi, Y.; OKa, M. \& Ishibashi, N. 1996. Preliminary observations on development of Pacific bluefin tuna Thunnus thynnus (Scombridae) larvae reared in the laboratory, with special reference to the digestive system. Marine \& Freshwater Resource 47(2):261-269.

Kendall, A. W.; Ahlstrom, E. H. \& Moser, H. G. 1984. Ontogeny and systematics of fishes. Lawrence, American Society Icthyologists and Herpetologists. p.11-12.

Koumoundouros, G.; Divanach, P. \& Kentouri, M. 1999. Ontogeny and allometric plasticity of Dentex dentex (Osteichthyes: Sparidae) in rearing conditions. Marine Biology 135:561-572.

Landines, M. A.; Senhorini, J. A.; Sanabria, A. I. \& Urbinati, E. C. 2003. Desenvolvimento embrionário do pintado (Pseudoplatystoma coruscans Agassiz, 1829). Boletim Técnico do CEPTA 16:1-13.

Leis, J. M. \& TRnSKI, T. 1989. The larvae of Indo-Pacific shore fishes. Honolulu, University of Hawaii Press. 371p.

Lima, A. R. A.; Barletta, M.; Dantas, D. V.; Possato, F. E.; Ramos, J. A. A.; Costa, M. F. 2012. Early development and allometric shifts during the ontogeny of a marine catfish (Cathorops spixii-Ariidae). Journal of Applied Ichthyology 28:217-225.

Luz, R. K.; Reynalte-Tataje, D. A.; Ferreira, A. A. \& Zaniboni-Filho, E. 2001. Desenvolvimento embrionário e estágios larvais do mandiamarelo Pimelodus maculatus. Boletim do Instituto de Pesca 27(1):49-55.

Maciel, C. M. R. R.; Júnior, A. M.; Donzele, J. L.; Lanna, E. A. T. \& Menin, E. 2009. Desenvolvimento morfológico das larvas de Hoplias lacerdae Miranda Ribeiro, 1908 (Characiformes, Erythrinidae), da eclosão até a metamorfose, relacionado com a capacidade de capturar alimento exógeno. Revista Biotemas 22(3):103-111.

Makrakis, M. C.; Miranda, L. E.; Makrakis, S.; Junior, H. M. F.; Morlis, W. G.; Dias, J. H. P. \& Garcia, O. J. 2012. Diversity in migratory patterns among neotropical fishes in a highly regulated river basin. Journal of Fish Biology 81:866-881. 
Meijide, F. J. \& Guerrero, G. A. 2000. Embryonic and larval development of a substrate-brooding cichlid Cichlasoma dimerus Heckel, 1940 under laboratory conditions. Journal of Zoology 252:481-493.

Nakatani, K.; Baumgartner, G. \& Latini, J. D.1998. Descrição morfológica de larvas do mapará Hypophthalmus edentatus (Spix) (Osteichthyes, Hypophthalmidae) no reservatório de Itaipu (rio Paraná, Brasil). Revista Brasileira de Zoologia 15(3):687-696.

Nakatani, K.; Baumgartner, G. \& Tenório-Baumgartner, M. S. 1997. Larval development of Plagioscion squamosissimus (Heckel) (Perciformes, Sciaenidae) of Itaipu reservoir (Paraná river, Brazil). Revista Brasileira de Zoologia 14(1):35-44.

Nakatani, K.; Agostinho, A. A.; Bialetzki, A.; Baumgartner, G.; Sanches, P. V.; Makrakis, M. \& Pavanelli, C. S. 2001. Ovos e larvas de peixes de água doce: desenvolvimento manual de identificação de ovos e larvas de peixes brasileiros de água doce. Maringá, EDUEM. 378p.

Nascimento, F. L. \& Araujo-Lima, C. A. R. M. 1993. Descrição de larvas de Psectrogaster amazonica e Potamorhina altamazonica (Curimatidae, Pisces) da Amazonia Central. Acta Amazonica 23(4):457-472.

Oliveira, F. G. D.; Bialetzki, A.; Gomes, L. C.; Santin, M. \& Taguti, T. L. 2012. Larval development of Brycon hilarii (Characiformes, Characidae). Iheringia, Série Zoologia 102(1):62-70.

Osse, J. W. M. \& van den Boogaart, G. M. J. 1995. Fish larvae, development, allometric growth and the aquatic environment. ICES Marine Science Symposia 201:21-34.

. 2004. Allometric Growth in fish Larvae: Timing and Function. In: GovonI, J. J. ed. The development of form and function in fish and the question of larval adaptation. American Fisheries Society. Symposium, 40, Bethesda, Maryland. p.167-194.

Penha, J. M. F.; Mateus, L. A. F. \& Barbieri, G. 2004. Age and growth of the Pothole Shovelnose Catfish (Hemisorubim platyrhynchos) in the Pantanal. Brazilian Journal of Biology 64(4):833-840.

Peña, R.; Dumas, S. \& Rodríguez-Jaramillo, C. 2009. Development and distribution of intestinal enzymatic activity in Paralabrax maculatofasciatus (Steindachner, 1868) larvae fed live prey. Aquaculture Research 40:218-224.

Reis, R. E.; Kullender, S. O. \& Ferraris Jr, C. J. 2003. Check list of the freshwater fishes of South and Central America. Porto Alegre,
EDIPUCRS. 729p.

Reynalte-Tataje, D.; Zaniboni-Filho, E. \& Esquivel, J. R. 2004. Embryonic and larvae development of piracanjuba, Brycon orbignyanus Valenciennes, 1849 (Pisces, Characidae). Acta Scientiarum 26(1):67-71.

Reynalte-Tataje, D. A.; Zaniboni-Filho, E. \& Muelbert, B. 2001. Stage of the embryonic development of the piavuçu Leporinus macrocephalus (Garavello \& Britski, 1988). Acta Scientarium 23(4):823-827.

Ribeiro, C.; Leme dos Santos, H. \& Bolzan, A. 1995. Estudo comparativo da embriogênesis de peixes ósseos (pacu, Piaractus mesopotamicus; tambaqui, Colossoma macropomum e híbrido tambacu). Revista Brasileira de Biologia 55(1):65-68.

Sanches, P. V.; NaKatani, K. \& BialetzKi, A. 1999. Morphological description of the developmental stages of Parauchenipterus galeatus (Linnaeus, 1766) (Siluriformes, Auchenipteridae) on the floodplain of the upper Paraná River. Revista Brasileira de Biologia 59(3):429438.

Sanches, P. V.; Baumgartner, G.; Bialetzki, A.; Siuberto, M. R.; Gomes, F. D. C; Nakatani, K. \& Barbosa, N. D. C. 2001. Caracterização do desenvolvimento inicial de Leporinus friderici (Osteichthyes, Anostomidae) da bacia do rio Paraná. Acta Scientiarum 23(2):383389.

SNYDER, D. E. 1979. Myomere and vertebrae counts of the North America cyprinids and catostomids. In: НоYT, R. D. ed. Proceedings of Third Symposium on Larval Fish. Western Kentucky University, Bowling Gree. p. 53-69.

Sousa, W. T. Z. \& Severi, W. 2002. Desenvolvimento inicial de larvas de Rhaphiodon vulpinus Agassiz, 1829 (Characiformes, Cynodontidae). Revista Brasileira de Zoologia 19(1):85-94.

Suzuki, H. I.; Vazzoler, A. E. A. M.; Marques, E. E.; Lizama, M. A. P. $\&$ InADA, P. 2004. Reproductive ecology of the fish assemblages. In: Thomaz, S. M.; Agostinho, A. A. \& Hahn, N. S. eds. The upper Paraná River and its floodplain: physical aspects, ecology and conservation. Leiden, Backhuys Publishers. p. 271-291.

Van SNIK, G. M. J.; van den Boogaart, J. G. M. \& Osse, J. W. M. 1997. Larval growth patterns in Cyprinus carpio and Clarias gariepinus with attention to the finfold. Journal of Fish Biology 50:1339-1352.

Recebido em 31 de outubro de 2013. Aceito em 14 de março de 2014. ISSN 0073-4721

Artigo disponível em: www.scielo.br/isz 